\title{
Article \\ Super-Accuracy Calculation for the Half Width of a Voigt Profile
}

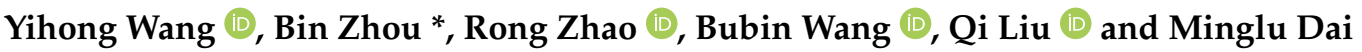

\author{
School of Energy and Environment, Southeast University, Nanjing 210096, China; wyh@seu.edu.cn (Y.W.); \\ zhaorong@seu.edu.cn (R.Z.); wbb@seu.edu.cn (B.W.); liuqi667@seu.edu.cn (Q.L.); 230208513@seu.edu.cn (M.D.) \\ * Correspondence: zhoubinde@seu.edu.cn
}

check for updates

Citation: Wang, Y.; Zhou, B.; Zhao, R.; Wang, B.; Liu, Q.; Dai, M. Super-Accuracy Calculation for the Half Width of a Voigt Profile. Mathematics 2022, 10, 210. https:// doi.org/10.3390/math10020210

Academic Editors: Sanjar Abrarov and Rehan Siddiqui

Received: 15 December 2021

Accepted: 8 January 2022

Published: 11 January 2022

Publisher's Note: MDPI stays neutral with regard to jurisdictional claims in published maps and institutional affiliations.

Copyright: (C) 2022 by the authors. Licensee MDPI, Basel, Switzerland. This article is an open access article distributed under the terms and conditions of the Creative Commons Attribution (CC BY) license (https:// creativecommons.org/licenses/by/ $4.0 /)$.

\begin{abstract}
A simple approximation scheme to describe the half width of the Voigt profile as a function of the relative contributions of Gaussian and Lorentzian broadening is presented. The proposed approximation scheme is highly accurate and provides an accuracy better than $10^{-17}$ for arbitrary $\alpha_{L} / \alpha_{G}$ ratios. In particular, the accuracy reaches an astonishing $10^{-34}$ (quadruple precision) in the domain $0 \leq \alpha_{L} / \alpha_{G} \leq 0.2371 \cup \alpha_{L} / \alpha_{G} \geq 33.8786$.
\end{abstract}

Keywords: Voigt profile; half width; super-accurate calculation

\section{Introduction}

The Voigt profile is widely used in optics, laser physics, plasma, astrophysics and spectroscopy [1-6]. By the convolution of both the Lorentzian and Gaussian profiles, the Voigt profile blends the physical characteristics of both profiles. Whereas the Lorentzian is associated with lifetime effects, the Gaussian is associated with thermal and statistical effects $[7,8]$. Accurate calculations and approximations for the Voigt spectral quantities in terms of the Gaussian and Lorentzian admixture are essential for the quantification of the profile. In actual application, the half width at half maximum (HWHM) of the Voigt profile, $\alpha_{V}$, is important [8-12]. Unfortunately, there is no analytically exact expression to describe the HWHM of the Voigt profile as a function of the HWHMs of the Lorentzian and Gaussian profiles, $\alpha_{L}$ and $\alpha_{G}$, respectively, and thus many approximations have been presented in the past to find simple relationships, i.e., composed of basic elementary functions only, between $\alpha_{V}, \alpha_{L}$ and $\alpha_{G}$ [13-17].

The approximation expression developed by Olivero and Longbothum (Equation (5) in [17]), to the best of the authors' knowledge, is the most accurate, and its estimation error is less than $0.01 \%$. Although this approximation is sufficient for the most practical tasks, the more accurate approximation may also be required in modern precision spectroscopy [18] Several highly accurate codes, such as the ACM Algorithm $680[19,20]$ (14 significant digits stated accuracy) and ACM Algorithm 916 [21] (20 significant digits) or arbitrary precision codes [22,23], are indispensable for evaluation of the Voigt profile, but not necessarily fast. However, in some cases, where we only focus on broadening [8-12,24], it is unwise to use these highly accurate codes. Therefore, a highly accurate approximate scheme specially used to evaluate the half width at half maximum of the Voigt profile is urgently needed.

In this work, we present a simple approximation scheme to describe the half width of the Voigt profile as a function of the relative contributions of Gaussian and Lorentzian broadening. The numerical calculations suggest that the proposed approximation expression can achieve super-accuracy calculation for Voigt profiles for arbitrary $\alpha_{L} / \alpha_{G}$ ratios. The rest of the paper is organized as follows: Section 2 details the methodology and derivation of the proposed approximation scheme. Error analysis and an applicable example for Dopplerbroadening thermometry (DBT) are carried out to validate the proposed approximation scheme in Sections 3 and 4, respectively. The paper is finally summarized in Section 5. 


\section{Methodology and Derivation}

The Voigt profile $g_{V}$ is the convolution of both the Lorentz profile $g_{L}$ and the Gaussian profile $g_{G}$ given by [25]

$$
g_{V}\left(v, \alpha_{L}, \alpha_{G}\right)=g_{L} \otimes g_{G}=\sqrt{\frac{\ln 2}{\pi}} \frac{1}{\alpha_{G}} K(x, y),
$$

where $\otimes$ is the convolution functional and $v$ is a generalized parameter for the wavenumber, frequency or photon energy. The Voigt function $K(x, y)$, normalized to $\sqrt{\pi}$,

$$
\begin{aligned}
K(x, y) & =\frac{y}{\pi} \int_{-\infty}^{+\infty} \frac{\mathrm{e}^{-t^{2}}}{(x-t)^{2}+y^{2}} d t \\
& =\operatorname{Re}\left[\mathrm{e}^{-z^{2}} \operatorname{erfc}(-\mathrm{i} z)\right]
\end{aligned}
$$

has been introduced for mathematical convenience. Here, the dimensionless variables $x=\sqrt{\ln 2}\left(v-v_{0}\right) / \alpha_{G}, y=\sqrt{\ln 2} \alpha_{L} / \alpha_{G}$ are a measure for the distance from the peak center $v_{0}$ and for the ratio of Lorentzian to Gaussian width, respectively, and $z=x+\mathrm{i} y$. At the line center $x=0$, the Voigt function can be expressed as the exponentially scaled complementary error function $K(0, y)=\exp \left(y^{2}\right) \operatorname{erfc}(y)$. Thus, the half-width scale in units of Gaussian broadening of a Voigt profile, $\Gamma$, for a given value of $y$ is given by the following equation:

$$
K(\Gamma, y)=\frac{1}{2} \mathrm{e}^{y^{2}} \operatorname{erfc}(y)
$$

where $\Gamma$ is defined as $\Gamma=\sqrt{\ln 2} \alpha_{V} / \alpha_{G}$. The variables $x$ and $y$ are more convenient for lines which are predominantly Gaussian while the variables $X=x / y$ and $\eta=1 / y$ are more convenient for lines which are predominantly Lorentz [26]. Thus, the half-width scale in units of Lorentzian broadening of a Voigt profile, $\Gamma^{\prime}$, for a given value of $\eta$ is given by the following equation:

$$
K\left(\frac{\Gamma^{\prime}}{\eta}, \frac{1}{\eta}\right)=\frac{1}{2} \mathrm{e}^{\frac{1}{\eta^{2}}} \operatorname{erfc}\left(\frac{1}{\eta}\right),
$$

where $\Gamma^{\prime}$ is defined as $\Gamma^{\prime}=\alpha_{V} / \alpha_{L}$.

Considering the Gaussian limit $(y \rightarrow 0)$ and the Lorentz limit $(y \rightarrow \infty)$ of Equation (1) [18]

$$
\begin{aligned}
\lim _{y \rightarrow 0(\eta \rightarrow \infty)} g_{V}\left(v, \alpha_{L}, \alpha_{G}\right) & =g_{G}\left(v, \alpha_{G}\right)=\sqrt{\frac{\ln 2}{\pi}} \frac{1}{\alpha_{G}} e^{-x^{2}}, \\
\lim _{y \rightarrow \infty} g_{V \rightarrow 0)}\left(v, \alpha_{L}, \alpha_{G}\right) & =g_{L}\left(v, \alpha_{L}\right)=\frac{1}{\pi} \frac{1}{\alpha_{L}} \frac{1}{1+X^{2}},
\end{aligned}
$$

the following two half-width limits can be easily deduced

$$
\begin{gathered}
\Gamma \rightarrow \sqrt{\ln 2}(y \rightarrow 0), \\
\Gamma^{\prime} \rightarrow 1 \quad(y \rightarrow \infty) .
\end{gathered}
$$

\subsection{The Half Width of Voigt Profile with Small $y(0 \leq y \leq 0.6993)$}

Combining Equations (2) and (3), the following equation for the half-width scale in units of Gaussian broadening, $\Gamma$, can be obtained

$$
\mathrm{e}^{-\Gamma^{2}} \sin (2 \Gamma y) \operatorname{Im}[\operatorname{erfc}(y-\mathrm{i} \Gamma)]+\mathrm{e}^{-\Gamma^{2}} \cos (2 \Gamma y) \operatorname{Re}[\operatorname{erfc}(y-\mathrm{i} \Gamma)]=\frac{1}{2} \operatorname{erfc}(y) .
$$

Suppose that the half width $\Gamma$ of a Voigt profile with small $y$ can be expanded by the following power series

$$
\Gamma(y)=\sum_{n=0}^{\infty} p_{n} y^{n}
$$


By combining Equations (8) and (9) and using the method of equating coefficients, any of the coefficients $p_{n}$ in Equation (9) can be determined. In this work, we have derived the recurrence equation satisfied by the expression of the $(r+1)$-th coefficient $p_{r}(r \geq 1)$ of each order in Equation (9) as follows

$$
\begin{aligned}
p_{r}= & \frac{\mathrm{e}^{p_{0}{ }^{2}}}{-2 p_{0}}\left[s_{r}-q_{r} \exp \left(-p_{0}{ }^{2}\right)-\sum \quad \begin{array}{c}
j+k+m=r \\
0 \leq j \leq r-1,0 \leq k \leq r-1,1 \leq m \leq r
\end{array}\right. \\
& \left.-\sum_{k=1}^{r-1} q_{r-k} f_{k}-\sum_{n=2}^{r} \frac{\alpha_{n, r}, b_{n}}{n !}\right],
\end{aligned}
$$

where each coefficient in the above expression can be calculated by the first $r$ coefficients, $p_{0}, p_{1}, \ldots, p_{r-1}$. The derivation of Equation (10) and the definition of each coefficient can be found in Appendix A. We notice the fact that $\Gamma=\sqrt{\ln 2}$ at $y=0$, which indicates that the first coefficient $p_{0}=\sqrt{\ln 2}$. Therefore, the coefficient of each order in Equation (9) can be obtained immediately by solving the recurrence equation. For example, the first four coefficients are shown as follows

$$
\begin{aligned}
p_{0} & =\sqrt{\ln 2}, \\
p_{1} & =\operatorname{erfi}(\sqrt{\ln 2})-\frac{1}{\sqrt{\pi \ln 2}}, \\
p_{2} & =-\frac{\left(2 \theta_{1}^{2}-1\right) \theta_{3}^{2}}{2 \theta_{1}}-\frac{2 \theta_{1}^{4} \theta_{2}^{2}+6 \theta_{1}^{2}+1}{2 \theta_{1}^{3} \theta_{2}^{2}}+\frac{4 \theta_{3}}{\theta_{2}}, \\
p_{3} & =\frac{4\left(4 \theta_{1}^{2}-1\right) \theta_{3}}{\theta_{1}^{2} \theta_{2}^{2}}+\frac{2}{3}\left(2 \theta_{1}^{2}-3\right) \theta_{3}\left(\theta_{3}^{2}+1\right) \\
& -\frac{\theta_{1}^{2}\left(56 \theta_{1}^{2}+6\right)+3}{6 \theta_{1}^{5} \theta_{2}^{3}}-\frac{16 \theta_{1}^{4}\left(3 \theta_{3}^{2}+1\right)-2 \theta_{1}^{2}\left(21 \theta_{3}^{2}+4\right)-3 \theta_{3}^{2}}{6 \theta_{1}^{3} \theta_{2}},
\end{aligned}
$$

where constants $\theta_{1}=\sqrt{\ln 2}, \theta_{2}=\sqrt{\pi}$ and $\theta_{3}=\operatorname{erfi}(\sqrt{\ln 2})$, respectively, and $\operatorname{erfi}(z)=-\operatorname{ierf}(\mathrm{i} z)$ is the imaginary error function. In addition, the first 31 terms with 32 significant digits are given in Table A1.

\subsection{The Half Width of Voigt Profile with Large $y(y \geq 8.2507)$}

Numerical analysis, detailed in Appendix B, indicates that it is convenient to analyze the relationship between $\Gamma^{\prime 2}$ and $\eta$, and a simple series is given as following

$$
\begin{aligned}
\Gamma^{\prime 2}(\eta) & =1+\frac{3}{2} \eta^{2}-\frac{3}{2^{2}} \eta^{4}+\frac{15}{2^{3}} \eta^{6}-\frac{243}{2^{5}} \eta^{8}+\frac{2493}{2^{6}} \eta^{10}-\frac{927}{2^{2}} \eta^{12}+\frac{405783}{2^{8}} \eta^{14} \\
& -\frac{25390179}{2^{11}} \eta^{16}+\frac{446848569}{2^{12}} \eta^{18}-\frac{1089694161}{2^{10}} \eta^{20}+\frac{46704949839}{2^{12}} \eta^{22} \\
& -\frac{8735832539883}{2^{16}} \eta^{24}+\frac{221377058104455}{2^{17}} \eta^{26}-\frac{6044700753428715}{2^{18}} \eta^{28} \\
& +\frac{176555754371862947}{2^{19}} \eta^{30}-\ldots
\end{aligned}
$$

In the above expression, the coefficients of odd terms are all zero, and the even terms are all rational fractions, which are conducive to high-precision calculation.

\subsection{The Half Width of Voigt Profile with Middle $y(0.6993<y<8.2507)$}

The global nature of the Chebyshev approximation over finite intervals [27] overcomes the range limitations resulting from the asymptotic expansions of Equations (9) and (12). Because of the best uniform approximation property of the Chebyshev polynomial, the best uniform approximation expression of the Voigt profile with middle $y$ $(0.6993<y<8.2507)$ can be obtained. Numerical analysis suggests that it is useful to introduce the non-dimensional quantities $R=\alpha_{V} /\left(\alpha_{L}+\alpha_{G}\right)$ and $D=\left(\alpha_{L}-\alpha_{G}\right) /\left(\alpha_{L}+\alpha_{G}\right)=(y-$ $\sqrt{\ln 2}) /(y+\sqrt{\ln 2})$, and the best uniform approximation expression is given as following

$$
R(D)=\sum_{k=0}^{N} R\left(D_{k}\right) \prod_{0 \leq m \leq N} \leq \frac{D-D_{m}}{D_{k}-D_{m}}
$$


where $\left(D_{k}, R\left(D_{k}\right)\right)$ are a set of $N$ sample points without two identical points of $D_{k}$, and $D_{k}$ are given as following

$$
D_{k}=0.3648+0.4518 \cos \left(\frac{2 k-1}{2 n+2} \pi\right), k=1,2, \cdots, N+1 .
$$

For computational convenience, the above mathematical expression can be reformulated into the following equivalent form

$$
R(D)=\sum_{k=0}^{N} u_{k} D^{k},
$$

where the first 31 coefficients, $u_{k}$, with 32 significant digits are given in Table A3.

\subsection{Half Width Approximation Scheme for Arbitrary Voigt Profile}

In summary, two asymptotic expansions, Equations (9) and (12), are given for smaller $y$ and larger $y$, respectively, and a Chebyshev approximation, Equation (15), for middle $y$. Thus, the half-width approximation scheme, using the same variable $y$, for an arbitrary Voigt profile can be summarized as follows:

$$
\alpha_{V}(y) \approx\left\{\begin{array}{rr}
\frac{1}{\sqrt{\ln 2}} \Gamma(y) \alpha_{G} & \text { Equation (9) for } 0 \leq y \leq 0.6993 \\
R\left(\frac{y-\sqrt{\ln 2}}{y+\sqrt{\ln 2}}\right)\left(\alpha_{L}+\alpha_{G}\right) & \text { Equation (15) for 0.6993 }<y<8.2507, \\
\Gamma^{\prime}\left(\frac{1}{y}\right) \alpha_{L} & \text { Equation (12) for } y \geq 8.2507 .
\end{array}\right.
$$

It is worth pointing out that when the approximation scheme Equation (16) is implemented on a double precision platform, the accuracy is reduced due to the rounding errors. An improvement approximation scheme for double precision platforms is as follows:

$$
\alpha_{V}(y) \approx\left\{\begin{array}{rr}
\frac{1}{\sqrt{\ln 2}} \Gamma(y) \alpha_{G} & \text { Equation (9) for } 0 \leq y \leq 0.6993 \\
R\left(\frac{y-\sqrt{\ln 2}}{y+\sqrt{\ln 2}}\right)\left(\alpha_{L}+\alpha_{G}\right) & \text { Equation (15) for 0.6993 }<y<6.4196 \\
\Gamma^{\prime}\left(\frac{1}{y}\right) \alpha_{L} & \text { Equation (12) for } y \geq 6.4196 .
\end{array}\right.
$$

\section{Error Analysis}

Define the relative errors for the half width of Voigt profile in the form

$$
\Delta=\frac{\left[\alpha_{V}\right]_{\text {appr. }}-\left[\alpha_{V}\right]_{\text {ref. }}}{\left[\alpha_{V}\right]_{\text {ref. }}},
$$

where $\left[\alpha_{V}\right]_{\text {appr. }}$ is the approximate value given by Equation (16) or Equation (17) and $\left[\alpha_{V}\right]_{\text {ref. }}$. is the reference. The highly accurate reference values of the half width can be obtained by solving Equation (3) using the latest versions of MATLAB that supports the symbolic computing system with high number, i.e., 128 in this work, of significand precision.

Figure 1 shows (a) the comparison of each half-width functions and (b) the logarithm of relative error for the half width of the Voigt profile, respectively, in the $R, D$ format on a high-precision platform. As we can see, in the Gaussian dominant region, i.e., $0 \leq y \leq 0.6993(-1 \leq D \leq-0.0870)$, the approximation Equation (9) is highly accurate and provides an accuracy better than $10^{-17}$. Numerical results illustrate that the calculation accuracy of Equation (9) increases significantly with the decrease of $y$, which indicates that this expression is more suitable for the calculation of small $y$. In particular, the accuracy of Equation (9) can reach the super accuracy of $10^{-34}$ (quadruple precision) in the domain $0 \leq y \leq 0.1974(-1 \leq D \leq-0.6167)$. Similarly, in the Lorentz dominant region, i.e., $y \geq 8.2507(0.8167 \leq D \leq 1)$, the approximation Equation (12) is highly accurate and provides an accuracy better than $10^{-17}$. 

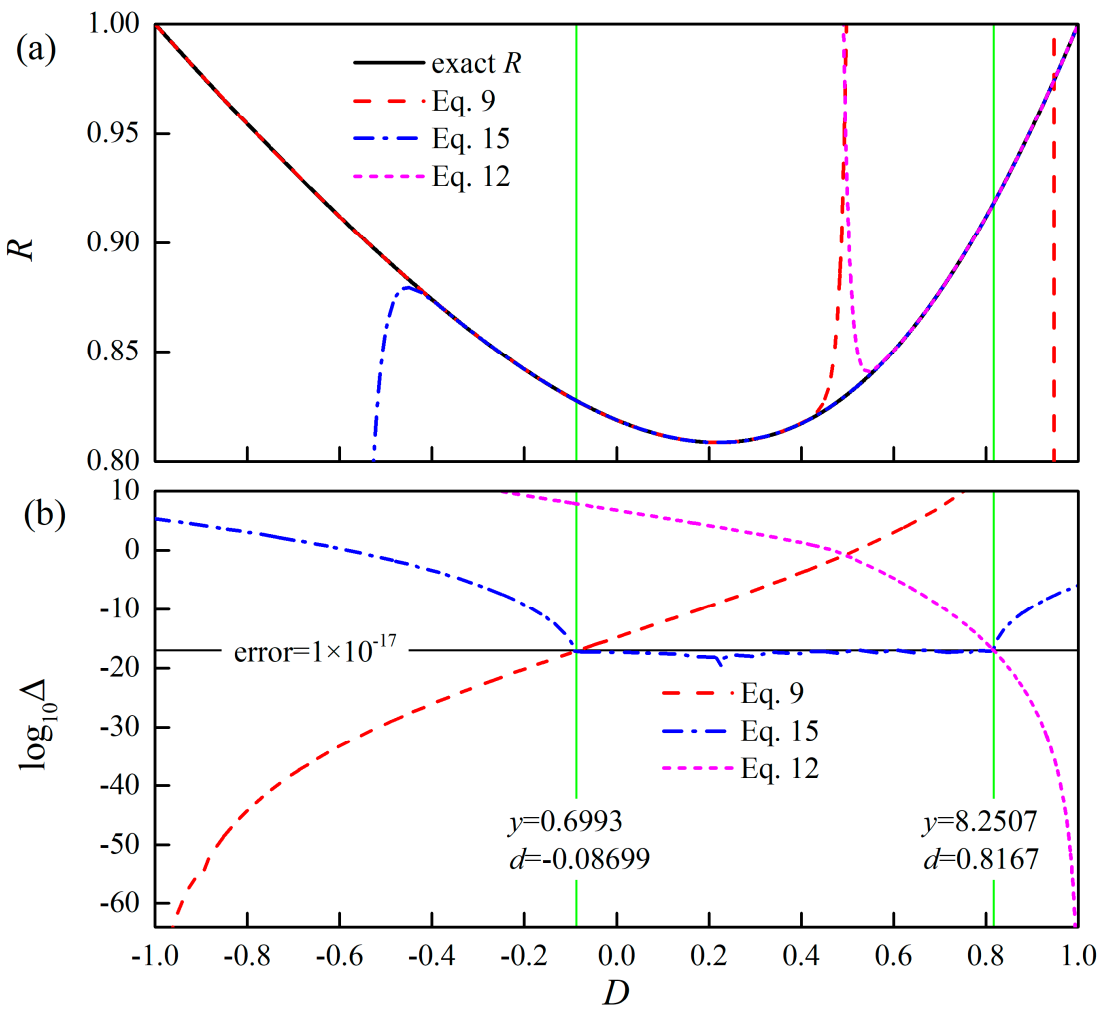

Figure 1. (a) The comparison of each half-width function and (b) the logarithm of relative error for the half width of the Voigt profile, respectively, in the $R, D$ format.

Numerical results show that the calculation accuracy of Equation (12) increases significantly with the increase of $y$, which indicates that this expression is more suitable for the calculation of large $y$. In particular, the accuracy of Equation (12) can reach the super- accuracy of $10^{-34}$ in the domain $y \geq 28.2058(0.9427 \leq D \leq 1)$. The Voigt width approximation is particularly important in the middle region, i.e., $0.6993<y<8.2507(-0.0870<D<0.8167)$, in which the width of the Gaussian profile is comparable to the width of the Lorentzian profile. Numerical results show that the approximation Equation (15) based on Chebyshev approximation with 30 degree is highly accurate and provides an accuracy better than $10^{-17}$ in the middle region. In summary, the approximation scheme Equation (16) is highly accurate and provides an accuracy better than $10^{-17}$ for arbitrary $\alpha_{L} / \alpha_{G}$ ratios, which indicates that the calculation error can be ignored for the general double-precision calculation platform (16 bits).

Furthermore, the accuracy of the proposed approximation scheme is compared with several commonly used approximation expressions [14-17] on a double precision platform. These approximate expressions are summarized in Table 1 . The relative errors of different approximation schemes on a double precision platform are shown in Figure 2. The maximum relative errors of Matveev, Thompson, Kielkopf and Olivero-Longbothum are $2.14 \times 10^{-2}, 4.32 \times 10^{-2}, 2.48 \times 10^{-4}$ and $2.37 \times 10^{-4}$, respectively. As a comparison, the calculation accuracy of the proposed approximation scheme is significantly improved, and its maximum relative error is $1.28 \times 10^{-14}$. 
Table 1. Empirical fit to the Voigt half width.

\begin{tabular}{cr}
\hline Author & Approximation Expression \\
\hline Matveev [14] & $\alpha_{V}(y)=\frac{1}{2 \sqrt{\ln 2}}\left[y+\sqrt{y^{2}+4 \ln 2}+0.1 y\left(1-\frac{2 y}{y+\sqrt{y^{2}+4 \ln 2}}\right)\right] \alpha_{G}$ \\
\hline Thompson et al. [16] & $\begin{array}{c}\alpha_{V}(y)=\frac{1}{\sqrt{\ln 2}}\left[(\ln 2)^{5 / 2}+(\ln 2)^{2} \cdot 2.69269 y+(\ln 2)^{3 / 2} \cdot 2.42843 y^{2}\right. \\
\left.+(\ln 2) \cdot 4.47163 y^{3}+(\ln 2)^{1 / 2} \cdot 0.07842 y^{4}+y^{5}\right]^{1 / 5} \alpha_{G}\end{array}$ \\
\hline Kielkopf [15] & $\alpha_{V}(y)=\frac{1}{2 \sqrt{\ln 2}}\left[(1+0.099 \ln 2) y+\sqrt{\left.(1-0.099 \ln 2)^{2} y^{2}+4 \ln 2\right] \alpha_{G}}\right.$ \\
\hline Olivero and Longbothum [17] & $\alpha_{V}(y)=\frac{1}{2 \sqrt{\ln 2}}\left(1.0692 y+\sqrt{0.86639 y^{2}+4 \ln 2}\right) \alpha_{G}$ \\
\hline
\end{tabular}

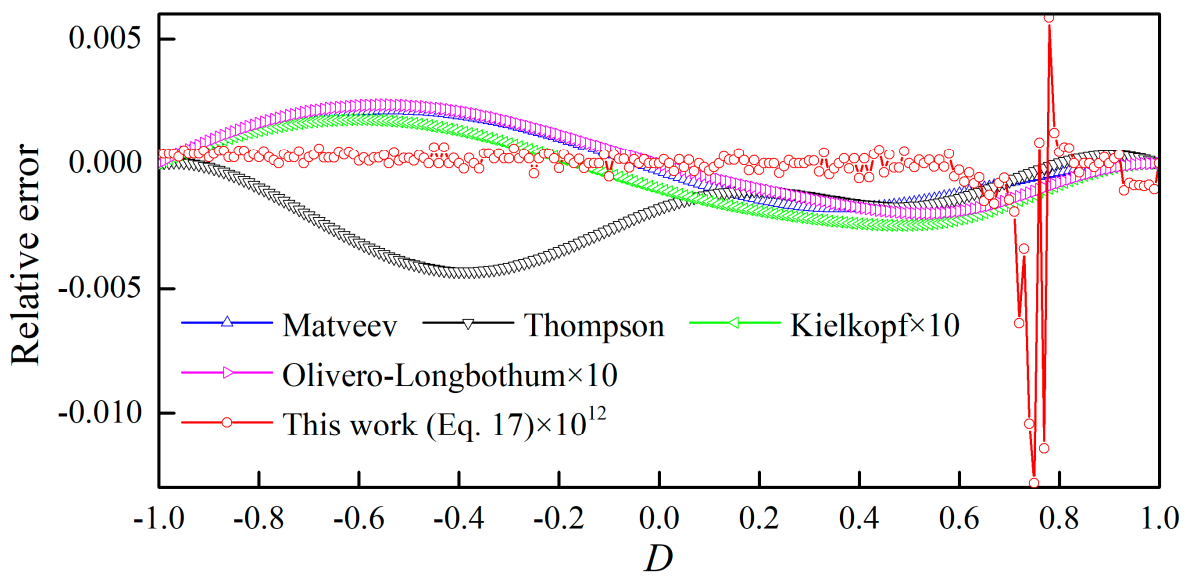

Figure 2. The relative errors of different approximation schemes on a double precision platform.

\section{An Applicable Example: Doppler-Broadening Thermometry (DBT)}

To evaluate the superiority of the proposed approximation scheme in terms of computational accuracy, a numerical simulation experiment of Doppler-broadening thermometry (DBT) using carbon dioxide $\left(\mathrm{CO}_{2}\right)$ transitions is presented in this section. The $\operatorname{Pe}(12)$ line of the $30012 \leftarrow 00001$ band of $\mathrm{CO}_{2}$, centered at $6337.990338 \mathrm{~cm}^{-1}$ with strength of $1.526 \times 10^{-23} \mathrm{~cm} /$ molecule [28], is used in this simulation. DBT is potentially an accurate and practical approach for thermodynamic temperature measurement $[12,24]$. DBT consists of retrieving the half width of Gaussian profile, $\alpha_{G}$, from the highly accurate observation of the shape of a given atomic or molecular line, in a laser-based absorption-spectroscopy experiment under a linear regime of radiation-matter interaction [12]. Once $\alpha_{G}$ is known, the thermodynamic temperature can be calculated as

$$
T=\frac{\alpha_{G}^{2} m c^{2}}{2 v_{0}^{2} k_{B} \ln 2}
$$

where $v_{0}=6337.990338 \mathrm{~cm}^{-1}$ is the central frequency, $k_{B}=1.38064852 \times 10^{-23} \mathrm{~J} / \mathrm{K}$ the Boltzmann constant, $c=2.99792485 \times 10^{8} \mathrm{~m} / \mathrm{s}$ the speed of light and $m=7.308032313279367 \times 10^{-26} \mathrm{~kg}$ the mass of $\mathrm{CO}_{2}$.

In general, the measured Voigt profile is deconvoluted to extract the Gaussian profile component, and then, the thermodynamic temperature is obtained by Equation (19). In this work, a simple deconvolution method based on the maximum and half width of the Voigt profile is used [29]. This method can easily calculate the half width of a Gaussian profile by solving the following non-linear system of equations

$$
\left\{\begin{array}{c}
g_{V, \max }=\sqrt{\frac{\ln 2}{\pi}} \frac{1}{\alpha_{G}} \exp \left(y^{2}\right)[1-\operatorname{erf}(y)], \\
\alpha_{V}=F\left(\alpha_{L}, \alpha_{G}\right),
\end{array}\right.
$$


where $g_{V, \max }$ is the measured maximum of the Voigt profile and $F\left(\alpha_{L}, \alpha_{G}\right)$ is some formula used for the half-width approximation. In this section, we only examine the numerical simulation results of the proposed approximation scheme Equation (17) and the OliveroLongbothum approximation.

The simulated Voigt profiles of $\mathrm{Pe}(12)$ line of $\mathrm{CO}_{2}$ at different pressures are shown in Figure 3. Obviously, the half width of the Voigt profile increases with higher pressure. This is because the Lorentzian broadening caused by molecular collisions becomes more and more significant as the pressure increases. Furthermore, the absolute deviations of DBT temperature calculations using different half-width approximations in the pressure range of $1 \mathrm{~Pa}$ to $2000 \mathrm{~Pa}$ are shown in Figure 4. Numerical simulation results demonstrate that the absolute temperature deviation is always less than $1.19 \times 10^{-12} \mathrm{~K}$ while the proposed approximation scheme Equation (17) is used for DBT temperature calculations. In contrast, the absolute temperature deviation becomes larger rapidly as the pressure increases for the Olivero-Longbothum approximation. The simulation experiments in this section reveal that a much more accurate half-width approximation scheme for an arbitrary Voigt profile is necessary when we expect to achieve high-accuracy temperature measurements at the $\mathrm{mK}$ level [12] using the Doppler-broadening thermometry method.

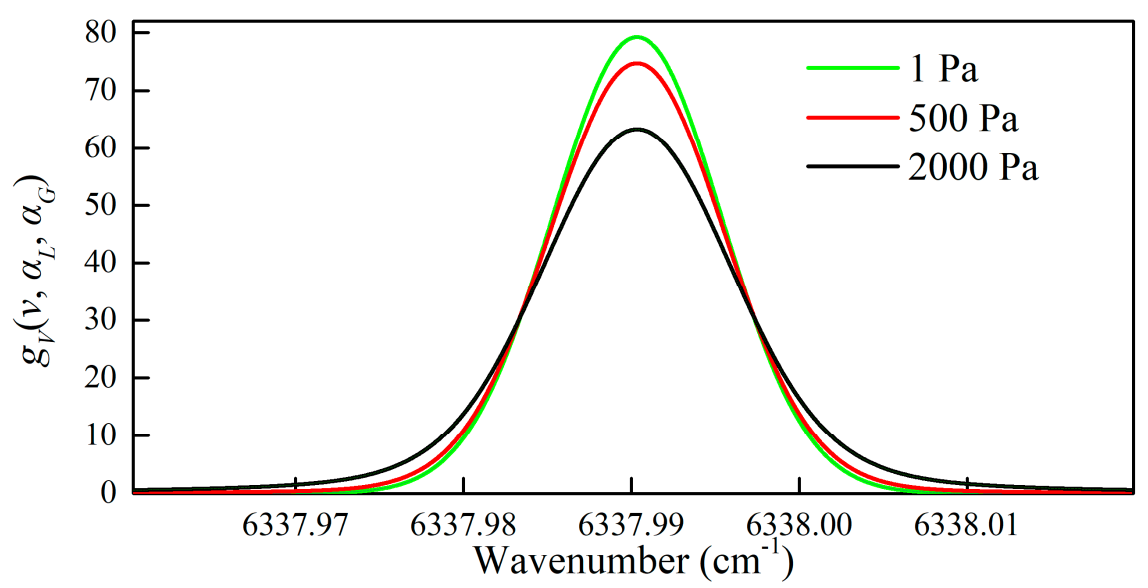

Figure 3. The simulated Voigt profile of $\mathrm{Pe}(12)$ line of $\mathrm{CO}_{2}$ at different pressures $\left(\mathrm{CO}_{2}\right.$ concentration is $1000 \mathrm{ppm}$, temperature is $300 \mathrm{~K}$ ).

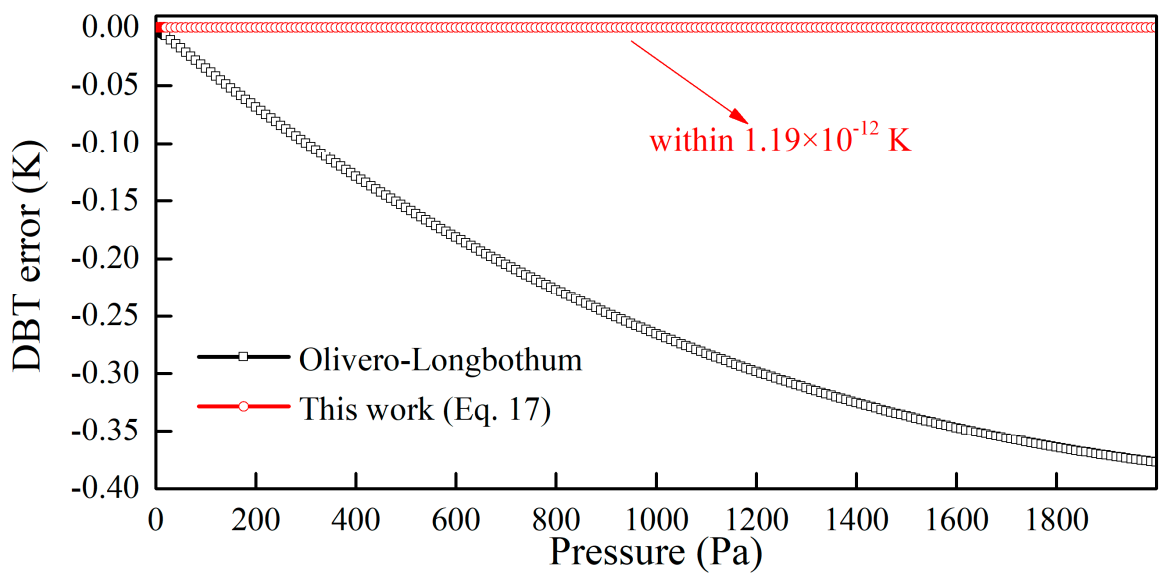

Figure 4. The absolute deviations of DBT temperature calculations using different half-width approximations. 


\section{Conclusions}

A simple approximation scheme to describe the half width of the Voigt profile as a function of the relative contributions of Gaussian and Lorentzian broadening is presented in this work. The numerical calculations suggest that the proposed approximation scheme can achieve super-accuracy (better than $10^{-17}$ ) calculation for Voigt profiles for arbitrary $\alpha_{L} / \alpha_{G}$ ratios. In particular, the accuracy reaches an astonishing $10^{-34}$ in the domain $0 \leq y \leq 0.1974 \cup y \geq 28.2058\left(0 \leq \alpha_{L} / \alpha_{G} \leq 0.2371 \cup \alpha_{L} / \alpha_{G} \geq 33.8786\right)$. Furthermore, the coefficients in Equation (12) are all simple rational fractions, and the law behind this expression is also an interesting mathematical problem worth exploring. To evaluate the superiority of the proposed approximation scheme in terms of computational accuracy, a numerical simulation experiments of Doppler-broadening thermometry using carbon dioxide transitions is presented in this work. In addition, three simplified MATLAB codes can be found at the MATLAB Central website: https:/ /www.mathworks.cn/matlabcentral/fileexchange/9528 3-highly-accurate-width-of-the-voigt-profile (accessed on 26 December 2021).

Author Contributions: Conceptualization, Y.W.; methodology, Y.W.; MATLAB code, Y.W., R.Z., B.W., Q.L. and M.D.; validation, Y.W. and R.Z.; formal analysis, Y.W.; investigation, Y.W. and B.Z.; writingoriginal draft preparation, Y.W.; writing-review and editing, B.Z.; visualization, Y.W., B.W., R.Z., Q.L. and M.D.; supervision, B.Z.; funding acquisition, B.Z. All authors have read and agreed to the published version of the manuscript.

Funding: This research was funded by National Key Research and Development Program of China, grant number 2017YFB0603204.

Institutional Review Board Statement: Not applicable.

Informed Consent Statement: Not applicable.

Data Availability Statement: Not applicable.

Conflicts of Interest: The authors declare no conflict of interest.

\section{Appendix A}

In this part, we derive the expression of the $(r+1)$-th coefficient $p_{r}$ from the first $r$ coefficients $p_{0}, p_{1}, \ldots, p_{r-1}(r>=1)$ using the series analysis method.

$$
\Gamma(y)=\sum_{n=0}^{r-1} p_{n} y^{n}+\sum_{n=r}^{\infty} p_{n} y^{n}
$$

Step 1: Series expansion for the factors in Equation (5) at $y=0$

(1) Series expansion for $\mathrm{e}^{\Gamma^{2}}$

The exponential function $\mathrm{e}^{\Gamma^{2}}$ can be expanded into the following series at $y=0$ :

$$
\mathrm{e}^{\Gamma^{2}}=\mathrm{e}^{p_{0}^{2}}+\sum_{n=1}^{\infty} \frac{a_{n}}{n !}\left(\Gamma-p_{0}\right)^{n}=\mathrm{e}^{p_{0}^{2}}+\sum_{n=1}^{r-1} \frac{a_{n}}{n !}\left(\sum_{k=1}^{r-1} p_{k} y^{k}\right)^{n}+\left(y^{r-1}\right),
$$

where the coefficients $a_{n}$ are defined as:

$$
a_{n}=\left.\frac{\mathrm{d}^{n}}{\mathrm{~d} t^{n}} \mathrm{e}^{t^{2}}\right|_{t=p_{0}}=\mathrm{e}^{p_{0}^{2}} \sum_{k=\left\lceil\frac{n-1}{2}\right\rceil}^{n} \sum_{j=0}^{\lfloor k-(n-1) / 2\rfloor} \frac{(-1)^{j} p_{0}^{2 k-n}(1-2 j+2 k-n)_{n}}{j !(-j+k) !},
$$

where $(.)_{n}$ is the Pochhammer symbol. According to the multinomial theorem, Equation (A3) can be rewritten as follows, in ascending order of the degree of $y$ :

$$
e^{\Gamma^{2}}=\sum_{k=0}^{r-1} d_{k} y^{k}+\left(y^{r-1}\right)
$$


where the coefficients $d_{k}$ are defined as:

$$
d_{k}= \begin{cases}\mathrm{e}^{p_{0}^{2}} & k=0 \\ \sum_{n=1}^{k} \frac{\alpha_{n, k, k} a_{n}}{n !} & k \geq 1,\end{cases}
$$

where the coefficients $\alpha_{n, k, j}$ are defined as:

$$
\begin{gathered}
\alpha_{n, k, j}=\sum \\
n_{1}+n_{2}+\cdots+n_{j}=n \quad \frac{n !}{n_{1} ! \cdots n_{j} !} p_{1}{ }^{n_{1}} \cdots p_{j}{ }^{n_{j}} . \\
\\
n_{1}+2 n_{2}+\cdots+j n_{j}=k \\
n_{1} \geq 0, \cdots, n_{j} \geq 0
\end{gathered}
$$

(2) Series expansion for $\mathrm{e}^{-\Gamma^{2}}$

In the same way, the exponential function $\mathrm{e}^{-\Gamma^{2}}$ can be expanded into the following series at $y=0$ :

$$
\mathrm{e}^{-\Gamma^{2}}=\sum_{k=0}^{r-1} f_{k} y^{k}+\left(p_{r} b_{1}+\sum_{n=2}^{r} \frac{\alpha_{n, r, r} b_{n}}{n !}\right) y^{r}+\left(y^{r}\right),
$$

where the coefficients $f_{k}$ are defined as:

$$
f_{k}= \begin{cases}\mathrm{e}^{-p_{0}^{2}} & k=0 \\ \sum_{n=1}^{k} \frac{\alpha_{n, k, k} b_{n}}{n !} & k \geq 1,\end{cases}
$$

where the coefficients $b_{n}$ are defined as:

$$
b_{n}=\left.\frac{\mathrm{d}^{n}}{\mathrm{~d} x^{n}} \mathrm{e}^{-x^{2}}\right|_{x=p_{0}}=2^{n} \mathrm{e}^{-p_{0}{ }^{2}}\left(-p_{0}\right)^{n} n ! \sum_{k=0}^{\lfloor n / 2\rfloor} \frac{(-4)^{-k} p_{0}-2 k}{k !(-2 k+n) !} .
$$

(3) Series expansion for $\sin (2 \Gamma y)$

In the same way, the sine function $\sin (2 \Gamma y)$ can be expanded into the following series at $y=0$ :

$$
\sin (2 \Gamma y)=\sum_{m=1}^{r} g_{m} y^{m}+o\left(y^{r}\right),
$$

where the coefficients $g_{m}$ are defined as:

$$
g_{m}=\sum_{k=0}^{\left\lfloor\frac{m-1}{2}\right\rfloor}(-1)^{k} \frac{\beta_{1+2 k, m, m-1}}{(1+2 k) !},
$$

where the coefficients $\beta_{n, k, j}$ are defined as:

$$
\beta_{n, k, j}=\sum \begin{aligned}
& n_{0}+n_{1}+\cdots+n_{j}=n \\
& n_{0}+2 n_{1}+\cdots+(j+1) n_{j}=k \\
& n_{0} \geq 0, \cdots, n_{j} \geq 0
\end{aligned} \quad \frac{n !}{n_{0} ! \cdots n_{r} !} 2^{n} p_{0}{ }^{n_{0}} \cdots p_{j}{ }^{n_{j}} .
$$

(4) Series expansion for $\cos (2 \Gamma y)$

In the same way, the $\operatorname{cosine}$ function $\cos (2 \Gamma y)$ can be expanded into the following series at $y=0$ :

$$
\cos (2 \Gamma y)=\sum_{m=0}^{r} h_{m} y^{m}+o\left(y^{r}\right),
$$

where the coefficients $h_{m}$ are defined as:

$$
h_{m}= \begin{cases}1 & m=0 \\ \sum_{k=1}^{\left\lfloor\frac{m}{2}\right\rfloor} \frac{(-1)^{k} \beta_{2 k, m, m-1}}{(2 k) !} & m \geq 1 .\end{cases}
$$


(5) Series expansion for erfi $(\Gamma)$

In the same way, the imaginary error function erfi $(\Gamma)$ can be expanded into the following series at $y=0$ :

$$
\operatorname{erfi}(\Gamma)=\operatorname{erfi}\left(p_{0}\right)+\sum_{k=1}^{r-1} \sum_{n=1}^{k} \frac{\alpha_{n, k, k} e_{n}}{n !} y^{k}+\left(y^{r-1}\right),
$$

where the coefficients $e_{n}$ are defined as:

$$
e_{n}=\left.\frac{\mathrm{d}^{n} \operatorname{erfi}(t)}{\mathrm{d} t^{n}}\right|_{t=p_{0}}=\frac{\mathrm{e}^{p_{0}^{2}}}{\sqrt{\pi}} \sum_{k=\left\lceil\frac{n-2}{2}\right\rceil}^{n-1} \frac{2^{2+2 k-n} p_{0}^{1+2 k-n}(2+2 k-n)_{2(-1-k+n)}}{(n-k-1) !} .
$$

(6) Series expansion for $\operatorname{erfc}(y-i \Gamma)$

The complementary error function $\operatorname{erfc}(y-\mathrm{i} \Gamma)$ can be expanded into the following series at $y=0$ :

$$
\begin{aligned}
& \operatorname{erfc}(y-\mathrm{i} \Gamma)=1+\operatorname{ierfi}(\Gamma) \\
& -\frac{2}{\sqrt{\pi}} \mathrm{e}^{\Gamma^{2}} \sum_{k=1}^{\infty} \sum_{j=\left\lceil\frac{k-1}{2}\right\rceil}^{k-1} \frac{(-\mathrm{i})^{1-k} 2^{1+2 j-k}(2+2 j-k)_{2(-1-j+k)} y^{k} \Gamma^{1+2 j-k}}{k !(-1-j+k) !},
\end{aligned}
$$

and the third term in the above formula can be reduced to the following formula:

$$
\begin{aligned}
& \left.-\frac{2}{\sqrt{\pi}} \mathrm{e}^{\Gamma^{2}} \sum_{k=1}^{\infty} \sum_{j=\left\lceil\frac{k-1}{2}\right\rceil}^{k-1}\right\rceil \frac{(-\mathbf{i})^{1-k} 2^{1+2 j-k}(2+2 j-k)_{2(-1-j+k)} y^{k} \Gamma^{1+2 j-k}}{k !(-1-j+k) !} \\
& =\mathrm{e}^{\Gamma^{2}}\left(\sum_{n=1}^{r} c_{n} y^{n}+\left(y^{r}\right)\right),
\end{aligned}
$$

where the coefficients $c_{n}$ are defined as:

$$
\begin{aligned}
c_{n} & =-\frac{2}{\sqrt{\pi}}\left(\frac{(-1)^{n+1}+1}{2} \frac{1_{n-1} \mathrm{i}^{1-n}}{n !((n-1) / 2) !}\right. \\
& \left.+\sum_{k=1}^{n} \sum^{k-1} \quad j=\left\lceil\frac{k-1}{2}\right\rceil \quad \chi_{1+2 j-k, n-k, n-k} \frac{(-\mathrm{i})^{1-k} 2^{1+2 j-k}(2+2 j-k)_{2(-1-j+k)}}{k !(-1-j+k) !}\right) \\
& \quad 1+2 j-k \geq 1
\end{aligned}
$$

where the coefficients $\gamma_{n, k, j}$ are defined as:

$$
\begin{aligned}
\gamma_{n, k, j}=\sum & n_{0}+n_{1}+\cdots+n_{j}=n \\
& n_{1}+2 n_{2}+\cdots+j n_{j}=k \\
& n_{0} \geq 0, \cdots, n_{j} \geq 0
\end{aligned}
$$

Considering the expansion series of Equations (A2) and (A18), the third term in Equation (A17) can be reduced to the following formula:

$$
\begin{aligned}
& -\frac{2}{\sqrt{\pi}} \mathrm{e}^{\Gamma^{2}} \sum_{k=1}^{\infty} \sum_{j=\left\lceil\frac{k-1}{2}\right\rceil}^{k-1} \frac{(-\mathbf{i})^{1-k} 2^{1+2 j-k}(2+2 j-k)_{2(-1-j+k)} y^{k} \Gamma^{1+2 j-k}}{k !(-1-j+k) !} \\
& =\sum_{m=1}^{r} \sum_{k=0}^{m-1} c_{m-k} d_{k} y^{m}+\left(y^{r}\right) .
\end{aligned}
$$

By substituting Equations (A15) and (A21) into Equation (A17), the expressions of real part and imaginary part of the complementary error function $\operatorname{erfc}(y-\mathrm{i} \Gamma)$ can be expanded into the following series:

$$
\begin{aligned}
& \operatorname{Im}[\operatorname{erfc}(y-\mathrm{i} \Gamma)]=\sum_{k=0}^{r-1} l_{k} y^{k}+\left(y^{r-1}\right), \\
& \operatorname{Re}[\operatorname{erfc}(y-\mathrm{i} \Gamma)]=\sum_{k=0}^{r-1} q_{k} y^{k}+\sum_{k=0}^{r-1} \operatorname{Re}\left[c_{r-k} d_{k}\right] y^{r}+o\left(y^{r}\right),
\end{aligned}
$$

respectively, where the coefficients $l_{k}$ and $q_{k}$ are defined as: 


$$
\begin{gathered}
l_{k}= \begin{cases}\operatorname{erfi}\left(p_{0}\right) & k=0 \\
\sum_{n=1}^{k} \frac{\alpha_{n, k, k} e_{n}}{n !}+\sum_{n=0}^{k-1} \operatorname{Im}\left[c_{k-n} d_{n}\right] & k \geq 1,\end{cases} \\
q_{k}= \begin{cases}1 & k=0 \\
\sum_{n=0}^{k-1} \operatorname{Re}\left[c_{k-n} d_{n}\right] & k \geq 1 .\end{cases}
\end{gathered}
$$

(7) Series expansion for $\operatorname{erfc}(y)$

The error function $\operatorname{erfc}(y)$ can be expanded into the following series at $y=0$ :

$$
\frac{1}{2} \operatorname{erfc}(y)=\sum_{n=0}^{r} s_{n} y^{n}+o\left(y^{r}\right),
$$

where the coefficients $s_{n}$ are defined as:

$$
s_{n}= \begin{cases}\frac{1}{2} & n=0 \\ -\frac{1+(-1)^{n+1}}{2} \frac{1}{\sqrt{\pi}} \frac{(-1)^{(n-1) / 2}}{((n-1) / 2) ! n} & n \geq 1 .\end{cases}
$$

\section{Step 2: Calculate $p_{r}$ by the method of comparing coefficients}

By substituting Equations (A7), (A10), (A13), (A22) and (A25) into Equation (8), the following expression is obtained:

$$
\begin{aligned}
& {\left[\sum_{k=0}^{r-1} f_{k} y^{k}\right]\left[\sum_{m=1}^{r} g_{m} y^{m}\right]\left[\sum_{k=0}^{r-1} l_{k} y^{k}\right]+\left[\sum_{k=0}^{r-1} f_{k} y^{k}+\left(p_{r} b_{1}+\sum_{n=2}^{r} \frac{\alpha_{n, r}, b_{n}}{n !}\right) y^{r}\right]} \\
& \times\left[\sum_{m=0}^{r} h_{m} y^{m}\right]\left[\sum_{k=0}^{r-1} q_{k} y^{k}+\sum_{k=0}^{r-1} \operatorname{Re}\left[c_{r-k} d_{k}\right] y^{r}\right]=\sum_{n=0}^{r} s_{n} y^{n}+\left(y^{r}\right)
\end{aligned}
$$

Therefore, a mathematical expression to determine the $(r+1)$-th coefficient $p_{r}$ is obtained by using the method of comparing coefficients:

$$
\begin{aligned}
& \sum_{j+k+m=r} \quad f_{j} g_{m} l_{k}+\sum_{j=1}^{r} \sum_{k=0}^{r-j} q_{r-j-k} f_{k} h_{j} \\
& \quad 0 \leq j \leq r-1,0 \leq k \leq r-1,1 \leq m \leq r \\
& +h_{0}\left[\sum_{k=1}^{r-1} q_{r-k} f_{k}+f_{0} \sum_{j=0}^{r-1} \operatorname{Re}\left[c_{r-j} d_{j}\right] y^{r}+\left(p_{r} b_{1}+\sum_{n=2}^{r} \alpha_{n, r, r} \frac{b_{n}}{n !}\right) q_{0}\right]=s_{r} .
\end{aligned}
$$

The recurrence equation satisfied by the expression of the $(r+1)$-th coefficient $p_{r}$ can be obtained by simplifying the above expression:

$$
\begin{aligned}
p_{r}=\frac{\mathrm{e}^{p_{0}}{ }^{2}}{-2 p_{0}}\left[s_{r}-q_{r} \exp \left(-p_{0}^{2}\right)-\sum \begin{array}{l}
j+k+m=r \\
\end{array}\right. & f_{j} g_{m} l_{k}-\sum_{j=1}^{r} \sum_{k=0}^{r-j} q_{r-j-k} f_{k} h_{j} \\
& \left.-\sum_{k=1}^{r-1} q_{r-k} f_{k}-\sum_{n=2}^{r} \frac{\alpha_{n, r}, b_{n}}{n !}\right] .
\end{aligned}
$$

Considering the initial value $p_{0}=\sqrt{\ln 2}$, arbitrary term coefficients $p_{n}$ can be calculated by using Equation (A29), and the first 31 coefficients $p_{n}$ with 32 significant digits are given in Table A1. 
Table A1. The first 31 coefficients $p_{n}$ in Equation (9) with 32 significant digits.

\begin{tabular}{cccc}
\hline$n$ & $p_{n}$ & $n$ & $p_{n}$ \\
\hline 0 & 0.8325546111576977563531646448952 & 16 & $2.5308903977393059634088084205148 \times 10^{-8}$ \\
1 & 0.53254711842961210323020845059416 & 17 & $-3.3104307709547517055285672959576 \times 10^{-8}$ \\
2 & $-6.3839925995348583105863651935208 \times 10^{-3}$ & 18 & $-1.1821070040002130133075915099552 \times 10^{-8}$ \\
3 & $-7.5882994178697868047017954181619 \times 10^{-3}$ & 19 & $5.0020607880755762331999675884955 \times 10^{-9}$ \\
4 & $7.5685451134845100193553849814044 \times 10^{-4}$ & 20 & $3.2040951850692659104678394048668 \times 10^{-9}$ \\
5 & $6.4174309726033170181322853645455 \times 10^{-4}$ & 21 & $-4.9276721508012916216290609360574 \times 10^{-10}$ \\
6 & $-1.0278614365257442345642575963235 \times 10^{-5}$ & 23 & $-7.1352246104725448681836423474852 \times 10^{-10}$ \\
7 & $-6.6864392638387619203117167133824 \times 10^{-5}$ & 24 & $-3.2407999521382539130974667691197 \times 10^{-11}$ \\
8 & $-1.8800729899141457354675112660009 \times 10^{-5}$ & 25 & $1.4010883014405512366881008147675 \times 10^{-10}$ \\
9 & $9.3901358253570724565409358708571 \times 10^{-6}$ & 26 & $3.3772678382804066494130831543588 \times 10^{-11}$ \\
10 & $5.4149990265667553408636905696295 \times 10^{-6}$ & 27 & $-2.3680267709485323621904030934022 \times 10^{-11}$ \\
11 & $-1.2862976252461744893956942201673 \times 10^{-6}$ & 28 & $-1.1462686830778835681784218983719 \times 10^{-11}$ \\
12 & $-1.0759168918380548822306060203341 \times 10^{-6}$ & 29 & $3.0039670445166124668988923778107 \times 10^{-12}$ \\
13 & $7.8733635964790862989086501951507 \times 10^{-8}$ & 30 & $2.9478889620399924642669859987364 \times 10^{-12}$ \\
14 & $1.9255725519174188542320412973488 \times 10^{-7}$ & & $-9.7467645599626148566298439388065 \times 10^{-14}$ \\
15 & & & \\
\hline
\end{tabular}

\section{Appendix B}

The relationship between $\Gamma^{2}$ and $\eta$ can be written in the form of Taylor series as follows:

$$
\Gamma^{\prime 2}(\eta)=\sum_{n=0}^{\infty} T_{n} \eta^{n}
$$

where $T_{0}=1$ and the coefficients $T_{n}(n>1)$ can be estimated by the following recurrence relations:

$$
T_{n}=\lim _{\eta \rightarrow 0^{+}} \frac{\Gamma^{\prime 2}(\eta)-\sum_{m=0}^{n-1} T_{m} \eta^{m}}{\eta^{n}} \approx \frac{\Gamma^{\prime 2}(\varepsilon)-\sum_{m=0}^{n-1} T_{m} \varepsilon^{m}}{\varepsilon^{n}} \triangleq T_{n}^{*}(0<\varepsilon \ll 1) .
$$

In this work, the parameter $\varepsilon$ in the above equation is set to $1 \times 10^{-20}$ and the benchmark value of $\Gamma^{\prime}(\varepsilon)$ is obtained according to Equation (3) by using the latest versions of MATLAB that supports the symbolic computing system with high number of significand precision. The coefficients $T_{n}$ and $T_{n}{ }^{*}$ of the first 31 terms are given in Table A2. Numerical calculation results suggest that the $T_{n}{ }^{*}$ value can be rewritten as the sum of a rational fraction and a fairly small remainder. Fortunately, the reduction in accuracy due to the elimination of the remainder is negligible.

\begin{tabular}{|c|c|c|c|c|c|}
\hline$n$ & $T_{n}^{*}$ & $T_{n}$ & $n$ & $T_{n}^{*}$ & $T_{n}$ \\
\hline 0 & / & 1 & 16 & $\approx-25390179 / 2^{11}+1.1 \times 10^{-35}$ & $-25390179 / 2^{11}$ \\
\hline 1 & $\approx 0+1.5 \times 10^{-20}$ & 0 & 17 & $\approx 0+1.1 \times 10^{-15}$ & 0 \\
\hline 2 & $\approx 3 / 2-7.5 \times 10^{-41}$ & $3 / 2$ & 18 & $\approx 446848569 / 2^{12}-1.1 \times 10^{-34}$ & $446848569 / 2^{12}$ \\
\hline 3 & $\approx 0-7.5 \times 10^{-21}$ & 0 & 19 & $\approx 0+-1.1 \times 10^{-14}$ & 0 \\
\hline 4 & $\approx-3 / 2^{2}+1.9 \times 10^{-40}$ & $-3 / 2^{2}$ & 20 & $\approx-1089694161 / 2^{10}+1.1 \times 10^{-33}$ & $-1089694161 / 2^{10}$ \\
\hline 5 & $\approx 0+1.9 \times 10^{-20}$ & 0 & 21 & $\approx 0+1.1 \times 10^{-13}$ & 0 \\
\hline 6 & $\approx 15 / 2^{3}-7.6 \times 10^{-40}$ & $15 / 2^{3}$ & 22 & $\approx 46704949839 / 2^{12}-1.3 \times 10^{-32}$ & $46704949839 / 2^{12}$ \\
\hline 7 & $\approx 0-7.6 \times 10^{-20}$ & 0 & 23 & $\approx 0-1.3 \times 10^{-12}$ & 0 \\
\hline 8 & $\approx-243 / 2^{5}+3.9 \times 10^{-39}$ & $-243 / 2^{5}$ & 24 & $\approx-8735832539883 / 2^{16}+1.7 \times 10^{-31}$ & $-8735832539883 / 2^{16}$ \\
\hline 9 & $\approx 0+3.9 \times 10^{-19}$ & 0 & 25 & $\approx 0+1.7 \times 10^{-11}$ & 0 \\
\hline 10 & $\approx 2493 / 2^{6}-2.3 \times 10^{-38}$ & $2493 / 2^{6}$ & 26 & $\approx 221377058104455 / 2^{17}-2.3 \times 10^{-30}$ & $221377058104455 / 2^{17}$ \\
\hline 11 & $\approx 0-2.3 \times 10^{-18}$ & 0 & 27 & $\approx 0-2.3 \times 10^{-10}$ & 0 \\
\hline 12 & $\approx-927 / 2^{2}+1.6 \times 10^{-37}$ & $-927 / 2^{2}$ & 28 & $\approx-6044700753428715 / 2^{18}+3.4 \times 10^{-29}$ & $-6044700753428715 / 2^{18}$ \\
\hline 13 & $\approx 0+1.6 \times 10^{-17}$ & 0 & 29 & $\approx 0+3.4 \times 10^{-9}$ & 0 \\
\hline 14 & $\approx 405783 / 2^{8}-1.2 \times 10^{-36}$ & $405783 / 2^{8}$ & 30 & $\approx 176955754371862947 / 2^{19}+3.9 \times 10^{-8}$ & $176955754371862947 / 2^{19}$ \\
\hline 15 & $\approx 0-1.2 \times 10^{-16}$ & 0 & & & \\
\hline
\end{tabular}

Table A2. The first 31 coefficients $T_{n}$ in Equation (12) 


\section{Appendix C}

Table A3. The first 31 coefficients un in Equation (15) with 32 significant digits.

\begin{tabular}{cccc}
\hline $\boldsymbol{n}$ & $\boldsymbol{u}_{\boldsymbol{n}}$ & $\boldsymbol{n}$ & $\boldsymbol{u}_{\boldsymbol{n}}$ \\
\hline 0 & 0.81879767981374096480451126966969 & 16 & -11.608186550060767559947858982236 \\
1 & -0.087358831239253690600565585478191 & 17 & 98.530866614251729915080851559823 \\
2 & 0.16111263881308988360982026625923 & 18 & -520.58001078415212154632736105054 \\
3 & 0.10352476879958392716101379868109 & 19 & 1996.0992356052342655084175033613 \\
4 & 0.044701941374241324794152587529398 & 20 & -5861.8655902675083091764869931816 \\
5 & -0.0014922440275783965022042298334427 & 21 & 13508.475417035315538373423012345 \\
6 & -0.025999766558392062049748550766996 & 22 & -24679.6185172226446663493302537093 \\
7 & -0.027433278283219905509735236616617 & 23 & 35800.136359576107078418696532107 \\
8 & -0.012324451041403454228824532372558 & 24 & -41003.1334660956855472430284467431 \\
9 & 0.0076580003679061144826236693340858 & 25 & 36602.695793508138571786897415062 \\
10 & 0.020609356479185309053858762785961 & 26 & -24908.669183448932665943004856528 \\
11 & 0.019910337726501870836014131102016 & 27 & 12466.93366679248692417862886569 \\
12 & 0.0077590409364772777791275317929576 & 28 & -4321.2090018088841034237110866818 \\
13 & -0.047206722791611042489905888231109 & 29 & 925.71414182435000624708620108582 \\
14 & 0.16487160565916573888004662456667 & 30 & -92.27479145146679165668461921968 \\
15 & 0.22613547743220062910838574670492 & & \\
\hline
\end{tabular}

\section{References}

1. Irmak, H. Various results for series expansions of the error functions with the complex variable and some of their implications. Turk. J. Math. 2020, 44, 1640-1648. [CrossRef]

2. Abrarov, S.M.; Quine, B.M.; Jagpal, R.K. A sampling-based approximation of the complex error function and its implementation without poles. Appl. Numer. Math. 2018, 129, 181-191. [CrossRef]

3. Abrarov, S.M.; Quine, B.M. A rational approximation for efficient computation of the Voigt function in quantitative spectroscopy. J. Math. Res. 2015, 7, 163-174. [CrossRef]

4. Wang, Y.; Zhou, B.; Liu, C. Calibration-free wavelength modulation spectroscopy based on even-order harmonics. Opt. Express 2021, 29, 26618-26633. [CrossRef] [PubMed]

5. $\quad$ Enemali, G.; Zhang, R.; Mccann, H.; Liu, C. Cost-Effective Quasi-Parallel Sensing Instrumentation for Industrial Chemical Species Tomography. IEEE Trans. Ind. Electron. 2021, 69, 2107-2116. [CrossRef]

6. Wang, Y.; Zhou, B.; Liu, C. Sensitivity and Accuracy Enhanced Wavelength Modulation Spectroscopy Based on PSD Analysis. IEEE Photonics Technol. Lett. 2021, 33, 1487-1490. [CrossRef]

7. Chen, M.; Meng, Z.; Wang, J.; Chen, W. Ultra-narrow linewidth measurement based on Voigt profile fitting. Opt. Express 2015, 23, 6803-6808. [CrossRef]

8. Selig, M.; Berghaeuser, G.; Raja, A.; Nagler, P.; Schueller, C.; Heinz, T.F.; Korn, T.; Chernikov, A.; Malic, E.; Knorr, A. Excitonic linewidth and coherence lifetime in monolayer transition metal dichalcogenides. Nat. Commun. 2016, 7, 3279. [CrossRef]

9. Zektzer, R.; Stern, L.; Mazurski, N.; Levy, U. Enhanced light-matter interactions in plasmonic-molecular gas hybrid system. Optica 2018, 5, 486-494. [CrossRef]

10. Antunes, B.; Velo-Antón, G.; Buckley, D.; Pereira, R.J.; Martínez-Solano, I. Characterization of an Atmospheric-Pressure Helium Plasma Generated by 2.45-GHz Microwave Power. Heredity 2012, 40, 3476-3481.

11. Hariri, A.; Sarikhani, S.J.O.A. Intrinsic linewidth calculation in an argon X-ray laser based on the model of geometrically dependent gain coefficient. Opt. Appl. 2017, 47, 325-335.

12. Gotti, R.; Moretti, L.; Gatti, D.; Castrillo, A.; Galzerano, G.; Laporta, P.; Gianfrani, L.; Marangoni, M. Cavity-ring-down Doppler-broadening primary thermometry. Phys. Rev. A 2018, 97, 012512. [CrossRef]

13. Whiting, E.E. An empirical approximation to the Voigt profile. J. Quant. Spectrosc. Radiat. Transf. 1968, 8, 1379-1384. [CrossRef]

14. Matveev, V.S. Approximate representations of absorption coefficient and equivalent widths of lines with voigt profile. J. Appl. Spectrosc. 1972, 16, 168-172. [CrossRef]

15. Kielkopf, J.F. New approximation to Voigt function with applications to spectral-line profile analysis. J. Opt. Soc. Am. 1973, 63, 987-995. [CrossRef]

16. Thompson, P.; Cox, D.E.; Hastings, J.B. Rietveld refinement of Debye-Scherrer synchrotron X-ray data from $\mathrm{Al}_{2} \mathrm{O}_{3}$. J. Appl. Crystallogr. 1987, 20, 79-83. [CrossRef]

17. Olivero, J.J.; Longbothum, R.L. Empirical fits to Voigt line width: Brief review. J. Quant. Spectrosc. Radiat. Transf. 1977, 17, 233-236. [CrossRef]

18. Rutkowski, L.; Masłowski, P.; Johansson, A.C.; Khodabakhsh, A.; Foltynowicz, A. Optical frequency comb Fourier transform spectroscopy with sub-nominal resolution and precision beyond the Voigt profile. J. Quant. Spectrosc. Radiat. Transf. 2018, 204, 63-73. [CrossRef] 
19. Poppe, G.P.M.; Wijers, C.M.J. More efficient computation of the complex error function. ACM Trans. Math. Softw. 1990, 16, 38-46. [CrossRef]

20. Poppe, G.P.M.; Wijers, C.M.J. Evaluation of the complex error function. ACM Trans. Math. Softw. 1990, 16, 47. [CrossRef]

21. Zaghloul, M.R.; Ali, A.N. Algorithm 916: Computing the Faddeyeva and Voigt Functions. ACM Trans. Math. Softw. 2011, 38, 1-22. [CrossRef]

22. Boyer, W.; Lynas-Gray, A.E. Evaluation of the Voigt function to arbitrary precision. Mon. Not. R. Astron. Soc. 2014, 444, 2555-2560. [CrossRef]

23. Molin, P. Multi-Precision Computation of the Complex Error Function. 2011. Available online: https://hal.archives-ouvertes.fr/ hal-00580855 (accessed on 26 December 2021).

24. Pan, Y.; Liao, W.; Wang, H.; Yao, Y.; Cai, J.; Qu, J. Cesium atomic Doppler broadening thermometry for room temperature measurement. Chin. Opt. Lett. 2019, 17, 060201. [CrossRef]

25. Schreier, F. Optimized implementations of rational approximations for the Voigt and complex error function. J. Quant. Spectrosc. Radiat. Transf. 2011, 112, 1010-1025. [CrossRef]

26. Armstrong, B.H. Spectrum line profiles: The Voigt function. J. Quant. Spectrosc. Radiat. Transf. 1967, 7, 61-88. [CrossRef]

27. Press, W.H.; Teukolsky, S.A.; Vetterling, W.T.; Flannery, B.P. Numerical Recipes with Source Code CD-ROM: The Art of Scientific Computing, 3rd ed.; Cambridge University Press: Cambridge, UK, 2007.

28. Gordon, I.E.; Rothman, L.S.; Hargreaves, R.J.; Hashemi, R.; Karlovets, E.V.; Skinner, F.M.; Conway, E.K.; Hill, C.; Kochanov, R.V.; Tan, Y.; et al. The HITRAN2020 molecular spectroscopic database. J. Quant. Spectrosc. Radiat. Transf. 2022, 277, 107949. [CrossRef]

29. Yin, Z.-Q.; Wu, C.; Gong, W.-Y.; Gong, Z.-K.; Wang, Y.-J. Voigt profile function and its maximum. Acta Phys. Sin. 2013, 62, 123301. (In Chinese) [CrossRef] 